\title{
A Novel Approach to Formulate and Optimize Orally Disintegrating Tablets of Bambuterol Hydrochloride
}

\author{
Liandong Hu*, Deliang $\mathrm{Gu}$, Qiaofeng Hu, Hailei Zhang and Xun Yang \\ College of Pharmacy, Hebei University and Key Laboratory of Pharmaceutical Quality Control of Hebei Province, Hebei University, Baoding, 071002, China
}

\begin{abstract}
The aim of this article was to choose the optimal ingredients for oral disintegrating tablets of bambuterol hydrochloride $(\mathrm{BBH})$ and use a novel approach to measure disintegration time. The disintegration time measured by a new approach was compared with disintegration time measured in oral cavity and conventional disintegration test. The result shows that the new apparatus is suitable for measuring the disintegration time. The effect of ingredients (diluents, disintegrants and adhesives) on the characteristics of the tablets was investigated. The orally disintegrating tablets of $\mathrm{BBH}$ were prepared by wet granulation. The results showed that the optimum proportion was $10 \mathrm{mg}$ $\mathrm{BBH}, 100 \mathrm{mg}$ lactose, $80 \mathrm{mg} \mathrm{MCC}, 10 \mathrm{mg}$ crospovidone (PVPP), $2 \mathrm{mg}$ aspartame, $2 \mathrm{mg}$ DL-malic acid. The orally disintegrating tablet disintegrated rapidly, tasted good and could be taken convenient.
\end{abstract}

Keywords: Orally disintegrating tablets (Odts); Bambuterol hydrochloride; Disintegration Time (DT); A New Apparatus; Formulation

\section{Introduction}

Because oflifestyle changes, a demand has arisen for the development of pharmaceutical forms that can be readily handled and taken by many patients. Rapidly disintegrating or dissolving drug delivery systems, in which drugs are administrated orally for direct ingestion without water, are particularly useful for geriatric, pediatric and traveling patients. These new forms of medicine are quite advantageous because they can be taken without any chewing or prior dispersion and/or dissolution processes [1-3]. Some of them (sublingual or buccal tablets) display a spontaneous disaggregation and fast absorption in the mouth upon contact with saliva [4]. Therefore, numerous studies on various compositions and manufacturing methods of orally disintegrating or dissolving tablets (ODTs) have been performed $[5,6]$.

DT is an important coefficient to orally disintegrating tablets. Compared with common tablet, ODTs disintegrate more quickly and need less solution. So the method to measure the common tablet disintegration time is not suitable for ODTs. At present, the USP, the $\mathrm{EP}$ and the $\mathrm{CP}$ are not established orally disintegrating tablet disintegration time method. Scholars have done many researches such as a modified dissolution test, fluid water [7] or a CCD camera [8] to obtain the scientific and reasonable method to measure DT. However, the proposed tests so far lack universality and convenience.

Bambuterol hydrochloride (BBH) is a direct acting sympathomimetic with predominantly-adrenergic activity ( $\beta_{2}$-agonist) [9]. It is an ester prodrug of $\beta_{2}$ adrenergic agonist terbutaline [10]. The dosage form of $\mathrm{BBH}$ is available in the market for the prophylaxis and treatment of chronic asthma and chronic bronchitis in pediatrics.

In this study we want to choose the optimal ingredients for oral disintegrating tablets of bambuterol hydrochloride (BBH) and use a novel approach to measure disintegration time.

\section{Experimental Materials}

BBH was obtained from Jiu Dian Pharmaceutical Co. Ltd (Hunan, People's Republc of China. MCC, PVP K30, PVPP were obtained from Shan He Excipents Co. Ltd (Anhui, People's Republic of China). Lactose was obtained from Kang Fu Biological Engineering Co. Ltd
(Zhenjiang, People's Republic of China). Mannitol was obtained from Hua Xu Pharmaceutical Co. Ltd (Hebei, People's Republic of China). Magnesium stearate was obtained from Ao Da Pharmaceutical Co. Ltd (Liaoning, People's Republic of China). All reagents were of analytical grade and used as received.

\section{Preparation of Tablets}

Tablets were manufactured by wet granulation (Figure 1). First, the $\mathrm{BBH}$ and the various quickly disintegrating tablet adjuvants (diluent, disintegrating agent and lubricant) were screened to produce a homogeneous mixture. The resulting mixtures were passed through a 100-mesh sieve and then granulated in a mortar using binder. The obtained granulates were dried in a $60^{\circ} \mathrm{C}$ oven (101-2A,Tianjin Taisite Instrument Co. Ltd, People's Republic of China) for 1 hour.

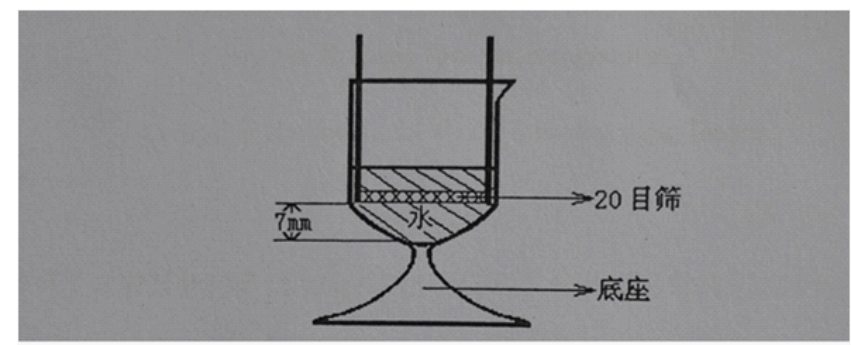

Figure 1: Apparatus of proposed new disintegration method for the vitro disintegration time determination.

*Corresponding author: Liandong Hu, College of Pharmacy, Hebei University and Key Laboratory of Pharmaceutical Quality Control of Hebei Province, Hebe University, Baoding, 071002, China, Tel: +86-312-5971107; Fax: +86-3125971107; E-mail: hbupharm@126.com

Received January 14, 2013; Accepted March 14, 2013; Published March 24 2013

Citation: Hu L, Gu D, Hu Q, Zhang H, Yang X (2013) A Novel Approach to Formulate and Optimize Orally Disintegrating Tablets of Bambuterol Hydrochloride. Pharmaceut Anal Acta 4: 216. doi:10.4172/2153-2435.1000216

Copyright: @ $2013 \mathrm{Hu}$ L, et al. This is an open-access article distributed unde the terms of the Creative Commons Attribution License, which permits unrestricted use, distribution, and reproduction in any medium, provided the original author and source are credited. 
The final granules were then passed through a 20 -mesh sieve. The filtered granules were directly compressed using a single-punch tablets formation machine (Shanghai Tianfan Pharmaceutical Machine Factory) fitted with a $9 \mathrm{~mm}$ diameter normal biconcave punch and die set. We can formulations in table 1.

\section{Tablet hardness}

The tablet hardness was determined using 10 random tablets using a hardness tester (PYC-A, Shanghai Huanghai Medical Instrument Factory, People's Republic of China).

\section{Friability test}

The tablet friability was measured as the percentage of weight loss of 30 tablets tumbled in a friability tester (CS-2, Tianjin Optical Instrument Factory, and People's Republic of China). After 4 min of rotation at $25 \mathrm{rpm}$, dust of tablets was removed and percentage weight loss was calculated.

\section{Measurement of disintegration time in new apparatus}

Figure 1 shows an illustration of the newly proposed disintegration method. Assessment of in vitro disintegration time was carried out using a sieve basket (The diameter of stainless stell tube was $25 \mathrm{~mm}$ and the height of it was $30 \mathrm{~mm}$ ).

The bottom of it was welded with 20 mesh stainless steel screen.), a beaker (Its upper inner diameter was slightly larger than the sieve basket's, and the lower was circular arc surface. The height of it was 7 $\mathrm{mm}$. It also had a base), a thermostat shaker (ZD-85A, Jintan Jieruier Electric Co. Ltd, People's Republic of China). $4 \mathrm{ml}$ of water maintained at $37^{\circ} \mathrm{C} \pm 0.5$ and stirred at $50 \mathrm{r} / \mathrm{min}$ was used as the disintegration. We measured six tablets for each formulation. The end point of disintegration was manifested as the disappearance of the last tablet fragment from the beaker. And the endpoint was measured visually using a stopwatch.

\section{Measurement of disintegration time in oral cavity}

Six healthy volunteers tested the complete DT of the tablets in oral cavity. Before the test, the mouth cavity was rinsed with a cup of water $(200 \mathrm{ml})$. The endpoint for the disintegration was the time when the tablet placed on the tongue had disintegrated until no lumps were remaining. The time required for the elimination of any residue or fragment of the tablets was measured with a stopwatch [11].

\section{Results and Discussion}

\section{Effect of diluent on disintegrating time}

Lactose, sucrose, mannitol and starch are used as diluents in ODTs. After reviewing some papers, we decided to use lactose, glucose, mannitol and sucrose as diluents in experiments. F1, F2, F3, F4 in table 1 shows that lactose is the best choice, for F1 disintegrates in 53s. F1 could be perfect compressed into tablets, and didn't have collapse phenomenon.

\section{Effect of disintegrating agent on disintegrating time}

Disintegrating agent is the key factor to influence DT. We always use CMS-Na, MCC, L-HPC and PVPP as disintegrating agent. Because MCC has porous structure and strong capillary effect, water can penetrate tablets and make it disintegrated. Due to its weak swelling property, it is often used with other disintegrating agents. Combination of MCC with PVPP was employed in this experiment using F1, F5, F6, F7 (MCC worked as variable, and PVPP remained the same amount) and F1, F8, F9, F10 (PVPP worked as variable, and MCC remained the same amount). Table 1 shows that DT is shorter, when we increase amount of MCC and PVPP. Because MCC tastes bad, and PVPP is expensive, we use F1 to reduce cost and ensure DT.

\section{Effect of the method of addition PVPP on disintegrating time}

In general, addition PVPP from inside and outside can shorten DT. To verify it, we design experiments using F1, F11, F12. From table 1 we can see that F11 has shorter DT than F1 and F12. So addition PVPP from inside and outside shortens DT.

\section{Effect of moistening agent to DT and granulation}

Moistening agent has an influence on granulation and DT, so it is necessary to screen a suitable moistening agent. We used distilled water, ethanol, $70 \%$ ethanol and 10\% PVP ethanol solution as moistening agent. The result is listed in table 2 .

Table 2 shows that ethanol is the best choice for moistening agent. Distilled water is not suitable, for BBH is good in water solubility. That makes granules bad. 70\% ethanol also makes granules bad, for there is water inside it. Then we used 10\%PVP ethanol to make granules better than using ethanol. But PVP is adhesives, so it makes DT longer than 120s. That's why we choose ethanol as moistening agent.

\section{Effect of taste masking on taste}

$\mathrm{BBH}$ needs to dissolve in orally, so the taste is important. $\mathrm{BBH}$

\begin{tabular}{|c|c|c|c|c|c|c|c|c|c|c|}
\hline Component & $\begin{array}{l}\text { BBH } \\
\text { (mg) }\end{array}$ & $\begin{array}{l}\text { MCC } \\
(\mathrm{mg})\end{array}$ & $\begin{array}{l}\text { PVPP } \\
\text { (mg) }\end{array}$ & $\begin{array}{l}\text { Lactose } \\
\text { (mg) }\end{array}$ & $\begin{array}{c}\text { Hydrated } \\
\text { mannitol (mg) }\end{array}$ & $\begin{array}{l}\text { Mannitol } \\
(\mathrm{mg})\end{array}$ & $\begin{array}{l}\text { Correctives } \\
\text { (mg) }\end{array}$ & $\begin{array}{l}\text { DT in the new } \\
\text { apparatus (s) }\end{array}$ & $\begin{array}{l}\text { DT in disintegration } \\
\text { apparatus (s) }\end{array}$ & $\begin{array}{l}\text { DT in oral } \\
\text { cavity (s) }\end{array}$ \\
\hline $\mathrm{F} 1$ & 10 & 80 & 10 & 100 & 0 & 0 & 10 & $53 \pm 2$ & $13 \pm 1$ & $62 \pm 8$ \\
\hline $\mathrm{F} 2$ & 10 & 80 & 10 & 0 & 100 & 0 & 10 & $90 \pm 3$ & $24 \pm 2$ & $110 \pm 10$ \\
\hline F3 & 10 & 180 & 10 & 0 & 0 & 0 & 10 & $>120$ & $38 \pm 3$ & $>120$ \\
\hline $\mathrm{F} 4$ & 10 & 80 & 10 & 0 & 0 & 100 & 10 & $70 \pm 3$ & $12 \pm 2$ & $83 \pm 9$ \\
\hline F5 & 10 & 0 & 10 & 180 & & & 10 & $>120$ & $40 \pm 3$ & $>120$ \\
\hline $\mathrm{F} 6$ & 10 & 60 & 10 & 120 & & & 10 & $65 \pm 3$ & $28 \pm 2$ & $82 \pm 9$ \\
\hline $\mathrm{F} 7$ & 10 & 100 & 10 & 80 & & & 10 & $50 \pm 2$ & $10 \pm 1$ & $62 \pm 8$ \\
\hline F8 & 10 & 80 & 8 & 102 & & & 10 & $65 \pm 3$ & $20 \pm 2$ & $77 \pm 10$ \\
\hline F9 & 10 & 80 & 0 & 110 & & & 10 & $>120$ & $37 \pm 2$ & $>120$ \\
\hline F10 & 10 & 80 & 12 & 98 & & & 10 & $45 \pm 2$ & $14 \pm 2$ & $60 \pm 9$ \\
\hline F11 & 10 & 80 & $\begin{array}{l}8 \text { inside, } \\
2 \text { outside }\end{array}$ & 100 & & & 10 & $48 \pm 2$ & $13 \pm 1$ & $58 \pm 9$ \\
\hline F12 & 10 & 80 & 0 & 100 & & & 10 & $>120$ & $43 \pm 4$ & $>120$ \\
\hline
\end{tabular}

Table 1: The formulations of orally disintegrating tablets of $\mathrm{BBH}$ 
Citation: Hụ L, Gu D, Hu Q, Zhang H, Yang X (2013) A Novel Approach to Formulate and Optimize Orally Disintegrating Tablets of Bambuterol Hydrochloride. Pharmaceut Anal Acta 4: 216. doi:10.4172/2153-2435.1000216

itself is bitter. That is why we need taste masking to improve the taste. According to the test result, BBH ODTs with aspartame and DL-malic acid can effectively improve the taste. And ten healthy volunteers feel no bitterness when they get BBH ODTs in their mouth.

\section{Effect of hardness on DT}

Tablet properties such as hardness are closely linked to causing DT. The preferable strength of the ODT is about $40 \mathrm{~N}$ and more preferably between 30 and $80 \mathrm{~N}$. Ten tablets from each of F1, F10, F11 were investigated in this test, for these formulations have good DT. The hardness of these tablets shows in table 3. From the results we can see that all these three formulations complied with regulation. When we take DT into account, F11 is the best choice.

\section{Friability test}

Tablet friability is a measurement of the tablet's physical strength. It was stated that an acceptable friability for tablets not exceeding $1 \%$. In friability test, we measured F1, F10, F11, for these formulation's DT are short. The results of friability test shown in table 4 . From table 4 we can see that F1, F10, F11 complied with the regulation as none of them had percentage loss in tablets' weights that exceeded $1 \%$, no tablets was cracked, split or broken in either formulation. Because F11 has shorter DT, it is the best choice.

\section{Evaluate of the novel method to measure DT}

The DT in the human sensory test is varied from table 1. From table 1, we can see that good correlation was obtained between the DT

\begin{tabular}{|c|c|c|}
\hline moistening agent & granulation & DT (s) \\
\hline distilled water (F11) & bad & - \\
\hline Ethanol (F11) & very good & 48 \\
\hline $70 \%$ ethnaol (F11) & bad & - \\
\hline $10 \%$ PVP ethanol (F11) & very good & $>120$ \\
\hline
\end{tabular}

Table 2: Effect of moistening agent on DT and granulation.

\begin{tabular}{|c|c|c|c|}
\hline Formulation & F1 & F10 & F11 \\
\hline No.1 (N) & 41 & 40 & 40 \\
\hline No.2 (N) & 40 & 41 & 40 \\
\hline No.3 (N) & 42 & 42 & 41 \\
\hline No.4 (N) & 41 & 41 & 42 \\
\hline No.5 (N) & 42 & 40 & 42 \\
\hline No.6 (N) & 41 & 42 & 41 \\
\hline No.7 (N) & 40 & 41 & 40 \\
\hline No.8 (N) & 41 & 42 & 41 \\
\hline No.9 (N) & 42 & 41 & 42 \\
\hline No.10 (N) & 41 & 40 & 41 \\
\hline
\end{tabular}

Table 3: The hardness of F1, F10, F11.

\begin{tabular}{|c|c|c|c|}
\hline Formulation & F1 & F10 & F11 \\
\hline weight before measured (g) & 6.012 & 6.008 & 5.997 \\
\hline weight after measured (g) & 5.994 & 5.978 & 5.961 \\
\hline lose weight (g) & 0.0018 & 0.030 & 0.036 \\
\hline lose weight\% & $0.3 \%$ & $0.5 \%$ & $0.6 \%$ \\
\hline $\begin{array}{c}\text { number of tablets with } \\
\text { cracked, split or broken }\end{array}$ & 0 & 0 & 0 \\
\hline
\end{tabular}

Table 4: Friability test of F1, F10, F11. determined in oral cavity and the new apparatus. The conventional disintegration test uses disintegration apparatus to determine ODTs DT, however it is doubtful whether it is suitable, for it requires a lot of water and shakes violently upper and lower. That makes ODTs disintegrate too fast to compare DT of different formulations. And we can see that DT determined by the conventional method does not have good correlation with DT determined in oral cavity. So a suitable disintegration test is needed. A critical factor might be the amount of water used in the test method, for the saliva volume is very small. The method we proposed utilizes only $4 \mathrm{ml}$ water to penetration of water. Another factor is mechanical vibration intensity of apparatus. The apparatus we used stir at $50 \mathrm{r} / \mathrm{min}$, so it shakes less intense than disintegration apparatus. From this point of view, our method also presents a clear advantage.

\section{Acknowledgements}

This work was supported by the Talent Introduction Program of Hebe University (No. y2005064) and by a grant of the Medical and Engineering Science Research Center of Hebei University (No. BM201109)

\section{References}

1. Chang RK, Guo X, Burnside B, Couch R (2000) Fast-dissolving tablets. Pharm Technol 24: 52-58.

2. Habib W, Khankari R, Hontz J (2000) Fast-dissolving drug delivery systems Crit Rev Ther Drug Carrier Syst 17: 61-72.

3. Fini A, Bergamante V, Ceschel GC, Ronchi C, de Moraes CA (2008) Fast dispersible/slow releasing ibuprofen tablets. Eur J Pharm Biopharm 69: 335341

4. Hisakadzu S, Yunxia B (2002) Preparation, evaluation and optimization of rapidly disintegrating tablets. Powder Technol 122: 188-198.

5. Mizumoto T, Masuda Y, Yamamoto T, Yonemochi E, Terada K (2005) Formulation design of a novel fast-disintegrating tablet. Int J Pharm 306: 83-90.

6. Fukami J, Yonemochi E, Yoshihashi Y, Terada K (2006) Evaluation of rapidly disintegrating tablets containing glycine and carboxy methyl cellulose. Int $J$ Pharm 310: 101-109.

7. Bi Y, Sunada K, Yonezawa Y, Danjo K, Otsuka A, et al. (1996) Preparation and evaluation of a compressed tablet rapidly disintegrating in the oral cavity. Chem Pharm Bull 44: 2121-2127.

8. Morita Y, Tsushima Y, Yasui M, Termoz R, Ajioka J, et al. (2002) Evaluation of the disintegration time of rapidly disintegrating tablets via a novel method utilizing a CCD camera. Chem Pharm Bull 50: 1181-1186.

9. Sweetman SC (ed.) Martindale: The Complete Drug Reference. 33rd ed Pharmaceutical Press, London 2002, pp.761.

10. Budavari S (ed.) The Merck Index (12thedn). Merck \& Co Inc, Whitehouse Station, NJ.1996, pp. 163.

11. Narazaki R, Harada T, Takami N, Kato Y, Ohwaki T (2004) A new method for disintegration studies of rapid disintegrating tablet. Chem Pharm Bull 52 704-707. 